\title{
Low circulating total adiponectin, especially its non-high-molecular weight fraction, represents a promising risk factor for colorectal cancer: a meta-analysis
}

This article was published in the following Dove Press journal: OncoTargets and Therapy

\author{
Weiqun $\mathrm{Lu}^{1,2}$ \\ Zhiliang Huang ${ }^{1,2}$ \\ Nan $\mathrm{Li}^{1,2}$ \\ Haiying Liu ${ }^{1,2}$ \\ 'Department of Gastrointestinal \\ Surgery, ${ }^{2}$ Guangzhou Key Laboratory \\ of Translational Medicine on Malignant \\ Tumor Treatment, Affiliated Cancer \\ Hospital \& Institute of Guangzhou \\ Medical University, Guangzhou, \\ People's Republic of China
}

\begin{abstract}
Aim: The principal goal of this meta-analysis is to test the hypothesis that circulating total adiponectin or certain fractions may represent a promising biological candidate in modulating the risk of colorectal cancer.

Methods: The processes of paper identification, paper selection and data extraction were accomplished independently by two authors. Effect-size estimates were expressed as weighted mean difference (WMD) and 95\% confidence interval (95\% CI). A total of 31 papers including 48 qualified studies (7,554 patients with colorectal cancer and 9,798 controls) were meta-analyzed.

Results: Pooling all studies found that circulating total adiponectin was significantly lower in patients with colorectal cancer than in controls (WMD: $-0.76 \mu \mathrm{g} / \mathrm{mL}, 95 \% \mathrm{CI}:-1.20$ to -0.32 , $p=0.001$ ), with significant heterogeneity $\left(I^{2}: 94.2 \%\right)$ and low publication bias (Egger's $p=0.336$ ). By adiponectin fractions, the difference in high-molecular weight (HMW) adiponectin was comparable between the two groups (WMD: $-0.22 \mu \mathrm{g} / \mathrm{mL}, 95 \% \mathrm{CI}:-0.70$ to $0.25, p=0.350$ ), while non-HMW adiponectin was significantly lower in patients with colorectal cancer than in controls (WMD: $-0.27 \mu \mathrm{g} / \mathrm{mL}, 95 \% \mathrm{CI}:-0.35$ to $-0.19, p<0.001)$, with marginal heterogeneity $\left(I^{2}: 52.3 \%\right)$. Subgroup analysis revealed that effect-size estimates were heterogeneous when grouping studies by cancer subtype, region, study design, matching status, gender and obesity. Further meta-regression analysis indicated that age and gender were significant potential sources of heterogeneity. The results showed the studied subgroups were not subject to publication bias (Egger's $p<0.1$ ).

Conclusion: Our data collectively indicate that low circulating total adiponectin, especially its non-HMW fraction, represents a promising risk factor for colorectal cancer. Further studies are needed to explore underlying mechanisms.
\end{abstract}

Keywords: colorectal cancer, total adiponectin, high-molecular weight adiponectin, non-highmolecular weight adiponectin, risk factor

\section{Introduction}

Adiponectin of molecular mass $28 \mathrm{kDa}$ is a member of the adipocytokines, and it is secreted exclusively by mature adipocytes. ${ }^{1}$ Biologically speaking, adiponectin possesses a well-defined set of properties, including insulin sensitization, anti-inflammation, anti-atherosclerosis, proapoptosis and antiproliferation. ${ }^{2,3}$ In circulation, adiponectin exists in forms of trimer, hexamer and high-molecular weight (HMW) complex, and these fractions are proven to have different biological activities. ${ }^{4}$ For example, HMW adiponectin has a close relationship to insulin sensitivity, while the relevance between non-HMW adiponectin and anti-inflammation is more obvious. ${ }^{5}$
Correspondence: Haiying Liu Department of Gastrointestinal Surgery, Affiliated Cancer Hospital \& Institute of Guangzhou Medical University, Hengzhigang Road 78, Yuexiu District, Guangzhou 510095 , Guangdong,

People's Republic of China

Tel/fax +862066671020

Email Ihy161226@163.com 
Mounting evidence from human studies reveal that low total adiponectin in circulation represents a predisposing status for development of colorectal cancer. ${ }^{6-8}$ By contrast, other researchers failed to confirm this claim, ${ }^{9-11}$ and some of them even found a higher concentration of circulating total adiponectin in patients with colorectal cancer than in controls. ${ }^{12,13}$ Several systematic reviews and meta-analyses have summarized published data involving the association between circulating total adiponectin and colorectal cancer risk, ${ }^{14-20}$ while interpretation of pooled findings in most cases is clouded by the presence of substantial heterogeneity and none considered adiponectin fractions as separate factors. With growing epidemiological data, our understanding on circulating adiponectin and its fractions and colorectal cancer needs replenishment and renewal. Hence, the principal goal of the present study is to test the hypothesis that circulating total adiponectin or certain fractions may represent a promising biological candidate in modulating the risk of colorectal cancer, assessed through a comprehensive meta-analysis. A secondary goal is to explore possible sources of heterogeneity of effect size across studies through both subgroup analysis and meta-regression analysis.

\section{Methods}

\section{Guideline}

The present study was carried out in strict accordance with the Preferred Reporting Items for Systematic Reviews and Meta-Analyses (PRISMA) guideline. ${ }^{21}$

\section{Paper identification}

Potentially eligible papers were identified through searching public databases of MEDLINE (PubMed), EMBASE, Web of Knowledge and Scholar (Google). All identified papers from each database were merged together and then duplicates removed. The key items used for paper identification included ("colorectal" or "colon" or "rectal") in title and ("cancer" or "carcinoma" or "tumor" or "tumour" or "neoplasm" or "adenoma") in title and ("adiponectin" or "adipocytokine" or "adipokine") in title/abstract. The process of paper identification was completed independently and in duplicate (Weiqun Lu and Zhiliang Huang) using the same key items mentioned above.

\section{Eligibility criteria}

Eligibility criteria consisted of both inclusion criteria and exclusion criteria. Papers were included only when the following conditions were met simultaneously: (i) publication using the English language; (ii) either cross-sectional or nested case-control study design; (iii) involvement of only human beings; and (iv) availability of circulating plasma or serum adiponectin concentration for patients with colorectal or colon or rectal cancer and cancer-free controls. Papers were excluded when any of the following conditions was true: (i) non-original contribution; (ii) narrative or systematic review or meta-analysis; (iii) conference abstract; and (iv) case report or case series.

\section{Paper selection}

Two authors (Weiqun Lu and Zhiliang Huang) selected the papers that met the above inclusion criteria through reviewing the title or abstract or the full text, if necessary, of each identified article. When disagreement happened during the selection process, it was resolved by discussion or seeking help from the third author (Haiying Liu).

\section{Data extraction}

From each qualified paper, data for analysis including, if available, the first author's name, publication year, country where the study was conducted, study design, cancer subtype, matching status, source of controls, selection of controls, sample size, assay method for circulating adiponectin, sample type, age, gender, body mass index (BMI), waist-hip ratio (WHR), waist circumference, physical activity, physical inactivity, smoking habit, family history, diabetes mellitus, glucose, insulin, C-reactive protein (CRP) and adiponectin were extracted and entered into databases by two authors (Weiqun Lu and Zhiliang Huang). Extracted data were compared, and disagreement was resolved through consensus and discussion.

\section{Statistical analysis}

Data were statistically analyzed by the Stata/SE software version 14.0 (StataCorp LP, College Station, TX, USA). Weighted mean difference (WMD) was adopted to assess the difference in circulating adiponectin concentration between patients and controls in both overall and subgroup analyses, and its $95 \%$ confidence interval $(95 \% \mathrm{CI})$ was estimated accordingly under the random-effects model based on the DerSimonian-Laird method. Heterogeneity was first evaluated with the $I^{2}$ statistic, and then was explored through both subgroup and meta-regression analyses. The $I^{2}$ statistic is expressed as a percentage figure, and the higher the figure, the more likely the heterogeneity becomes. It is widely accepted the significance cutoff point of $I^{2}$ statistic is set at $50 \%$. Publication bias was assessed by the Begg's funnel plot, filled funnel plot and Egger's regression asymmetry test. 
The Egger's regression asymmetry test records significance if the probability value is $<10 \%$.

\section{Results \\ Qualified studies}

A total of 339 papers were identified through searching public databases, and 295 of them were excluded after reading the title and abstract. Further, after full-text reviewing, 13 papers were excluded as they failed to meet our predefined inclusion criteria, leaving 31 eligible papers for meta-analysis in this study. ${ }^{5-13,22-43}$ Thereof, 16 papers provided data by cancer type, gender or race, and so there were 48 qualified studies.

\section{Study characteristics}

Table 1 shows the baseline characteristics of all qualified studies. After removing shared controls, this metaanalysis involved 7,554 patients with colorectal cancer and 9,798 controls. Of 48 qualified studies, only one study focused on HMW adiponectin. ${ }^{39}$ In addition, eight studies also measured HMW adiponectin, and non-HMW adiponectin was recorded in six studies.

\section{Overall analysis}

Overall analysis was based on 41 qualified studies, and the funnel plot illustrating the difference in total adiponectin concentrations between patients with colorectal cancer and controls is presented in Figure 1. Pooling effect size of all studies found that total adiponectin was significantly lower in patients with colorectal cancer than in controls (WMD: $-0.76 \mu \mathrm{g} / \mathrm{mL}, 95 \% \mathrm{CI}:-1.20$ to $-0.32, p=0.001$ ). However, this significant finding was clouded by strong evidence of statistical heterogeneity $\left(I^{2}: 94.2 \%, p<0.001\right)$.

Figure 2 shows another funnel plot quantifying the differences in adiponectin fractions - HMW adiponectin and non-HMW adiponectin - between patients with colorectal cancer and controls. The difference in HMW adiponectin was comparable between the two groups (WMD: $-0.22 \mu \mathrm{g} / \mathrm{mL}$, 95\% CI: -0.70 to $0.25, p=0.350$ ), with significant heterogeneity $\left(I^{2}: 97.3 \%, p<0.001\right)$. By contrast, non-HMW adiponectin was significantly lower in patients with colorectal cancer than in controls (WMD: $-0.27 \mu \mathrm{g} / \mathrm{mL}, 95 \% \mathrm{CI}:-0.35$ to $-0.19, p<0.001)$, with only marginal heterogeneity ( $\left.I^{2}: 52.3 \%, p=0.063\right)$.

\section{Subgroup analysis}

Table 2 summarizes the differences in total adiponectin between patients and controls by grouping studies according to cancer subtype, region, study design, matching status, sample source, gender, BMI, WHR and diabetes mellitus, respectively.

By cancer subtype, significant difference in total adiponectin was observed in patients with colorectal adenoma (CRA), colon cancer and rectum cancer, respectively, relative to controls (WMD: $-0.892,-1.073$ and $-0.681 \mu \mathrm{g} / \mathrm{mL}$, $p=0.010)$, with significant heterogeneity $\left(I^{2}: 80.1 \%, 97.3 \%\right.$ and $75.4 \%$ ).

By geographic region, besides Middle East, total adiponectin was significantly lower in patients than in controls from America, Europe and especially East Asia (WMD: $-1.707 \mu \mathrm{g} / \mathrm{mL}, p=0.008$ ), and only in America, there was no evidence of heterogeneity $\left(I^{2}: 36.7 \%\right)$.

By study design, significant difference was observed only in nested case-control studies (WMD: $-0.266 \mu \mathrm{g} / \mathrm{mL}$, $p<0.001)$, without heterogeneity $\left(I^{2}: 12.4 \%\right)$.

By matching status, studies with matched patients and controls failed to identify any significance (WMD: -0.099 $\mu \mathrm{g} / \mathrm{mL}, p=0.504)\left(I^{2}: 79.0 \%\right)$, while in studies with unclear matching status, there was significant difference in total adiponectin between patients and controls ( $\left.I^{2}: 96.1 \%\right)$.

By sample source, total adiponectin was significantly and comparably reduced in studies collecting plasma and serum samples for adiponectin measurement (WMD: -0.714 and $-0.803 \mu \mathrm{g} / \mathrm{mL}, p=0.030$ and $0.012, I^{2}: 95.1 \%$ and $93.3 \%$, respectively).

By sample size, grouping studies by median total sample size showed that the reduction of total adiponectin was obvious in studies with total sample size $>230$ (WMD: $-0.271 \mu \mathrm{g} / \mathrm{mL}, p<0.001$ ), and the likelihood of heterogeneity was low $(I: 12.1 \%)$.

By gender, total adiponectin was reduced significantly in male patients relative to male controls (WMD: $-0.671 \mu \mathrm{g} / \mathrm{mL}$, $p=0.025)\left(I^{2}: 90.4 \%\right)$, and there was no observable significance in females.

By obesity, studies were grouped by mean or median BMI at $25 \mathrm{~kg} / \mathrm{m}^{2}$, and significant reduction in total adiponectin was noted in both groups, especially in studies with BMI $<25 \mathrm{~kg} / \mathrm{m}^{2}$ (WMD: $-1.627 \mu \mathrm{g} / \mathrm{mL}, p=0.029$ ) ( $\left.I^{2}: 92.1 \%\right)$. In addition, when studies were grouped by mean or median WHR at $0.9 \mathrm{~m}$, there was significant reduction in studies with WHR $\geq 0.9 \mathrm{~m}$ (WMD: $-0.522 \mu \mathrm{g} / \mathrm{mL}$, $p=0.014$ ).

After restricting to studies involving subjects free of diabetes mellitus, the reduction in total adiponectin was not statistically significant, and there was significant heterogeneity. 
Table I The baseline characteristics of all qualified studies

\begin{tabular}{|c|c|c|c|c|c|c|c|}
\hline Study & Year & Region & $\begin{array}{l}\text { Cancer } \\
\text { subtype }\end{array}$ & Control features & $\begin{array}{l}\text { Study } \\
\text { design }\end{array}$ & $\begin{array}{l}\text { Matching } \\
\text { status }\end{array}$ & $\begin{array}{l}\text { ADI assay } \\
\text { (ELISA) }\end{array}$ \\
\hline Saetang et $\mathrm{al}^{25}$ (CRC) & 2016 & East Asia & CRC & W/o colonic polyps & Retrospective & NA & Thermo Fisher \\
\hline Saetang et $\mathrm{al}^{25}$ (colon) & 2016 & East Asia & Colon & W/o colonic polyps & Retrospective & NA & Thermo Fisher \\
\hline Saetang et $\mathrm{al}^{25}$ (rectum) & 2016 & East Asia & Rectum & W/o colonic polyps & Retrospective & NA & Thermo Fisher \\
\hline Inamura et $\mathrm{al}^{29}$ (HPFS) & 2016 & USA & CRC & W/o CRC & Nested & Yes & ALPCO \\
\hline Inamura et $\mathrm{al}^{29}$ (NHS) & 2016 & USA & CRC & W/o CRC & Nested & Yes & ALPCO \\
\hline Zekri et $\mathrm{al}^{13}$ & 2015 & Middle East & CRC & W/o CRC & Retrospective & NA & RayBiotech \\
\hline Demir et $\mathrm{al}^{37}$ & 2015 & Middle East & CRA & W/o CRC & Retrospective & NA & eBioscience \\
\hline Chandler et al'" & 2015 & USA & CRC & W/o CRC & Nested & Yes & ALPCO \\
\hline Ochs-Balcom et $\mathrm{al}^{26}$ (white) & 2014 & USA & CRA & W/o CRA & Nested & NA & $R \& D$ \\
\hline Ochs-Balcom et $\mathrm{al}^{26}(\mathrm{AA})$ & 2014 & USA & CRA & W/o CRA & Nested & NA & $\mathrm{R} \& \mathrm{D}$ \\
\hline Aleksandrova et $\mathrm{al}^{5}$ (colon) & 2014 & Europe & Colon & W/o CRC & Nested & Yes & ALPCO \\
\hline Aleksandrova et $\mathrm{al}^{5}$ (rectum) & 2014 & Europe & Rectum & W/o CRC & Nested & Yes & ALPCO \\
\hline Aleksandrova et $\mathrm{al}^{40}$ (men) & 2014 & Europe & Colon & W/o CRC & Nested & Yes & ALPCO \\
\hline Aleksandrova et al ${ }^{40}$ (women) & 2014 & Europe & Colon & W/o CRC & Nested & Yes & ALPCO \\
\hline Abe Vicente et $\mathrm{al}^{43}$ & 2014 & USA & CRC & Healthy & Retrospective & NA & $\mathrm{R} \& \mathrm{D}$ \\
\hline Song et $\mathrm{al}^{24}$ (men) & 2013 & USA & CRC & W/o CRC & Nested & Yes & Linco \\
\hline Song et $\mathrm{al}^{24}$ (women) & 2013 & USA & CRC & W/o CRC & Nested & Yes & Linco \\
\hline Danese et $\mathrm{al}^{38}$ (men) & 2013 & Europe & CRA & W/o CRC & Retrospective & Yes & Mediagnost \\
\hline Danese et $\mathrm{al}^{38}$ (women) & 2013 & Europe & CRA & W/o CRC & Retrospective & Yes & Mediagnost \\
\hline Kemik et $\mathrm{al}^{8}$ (colon) & 2012 & Middle East & Colon & Healthy & Retrospective & NA & Linco \\
\hline Kemik et al ${ }^{8}$ (rectum) & 2012 & Middle East & Rectum & Healthy & Retrospective & NA & Linco \\
\hline Ho et $\mathrm{al}^{30}$ & 2012 & USA & CRC & W/o CRC & Nested & NA & Millipore \\
\hline Hillenbrand et $\mathrm{al}^{31}$ (men) & 2012 & Europe & $\mathrm{CRC}$ & Healthy & Retrospective & NA & Millipore \\
\hline Hillenbrand et $\mathrm{al}^{31}$ (women) & 2012 & Europe & CRC & Healthy & Retrospective & NA & Millipore \\
\hline Gulcelik et $\mathrm{al}^{32}$ & 2012 & Middle East & Colon & W/o cancer & Retrospective & NA & B-Bridge \\
\hline Chen et $\mathrm{al}^{39}(\mathrm{E})$ & 2012 & East Asia & CRC & W/o colonic polyps & Retrospective & NA & Adlitteram \\
\hline Chen et $\mathrm{al}^{39}(\mathrm{~A})$ & 2012 & East Asia & CRC & W/o colonic polyps & Retrospective & NA & Adlitteram \\
\hline Aleksandrova et $\mathrm{al}^{4 l}$ (colon) & 2012 & Europe & Colon & W/o CRC & Nested & Yes & ALPCO \\
\hline Aleksandrova et al ${ }^{4 \mid}$ (rectum) & 2012 & Europe & Rectum & W/o CRC & Nested & Yes & ALPCO \\
\hline Al-Harithy and Al-Zahrani ${ }^{42}$ (men) & 2012 & Middle East & $\mathrm{CRC}$ & Healthy & Retrospective & Yes & ALPCO \\
\hline Al-Harithy and $\mathrm{Al}-\mathrm{Zahrani}{ }^{42}$ (women) & 2012 & Middle East & CRC & Healthy & Retrospective & Yes & ALPCO \\
\hline Gialamas et $\mathrm{al}^{34}$ & 2011 & Europe & CRC & Healthy & Retrospective & Yes & RI \\
\hline Chronis et al ${ }^{10}$ (NOAA) & 2011 & Europe & CRA & W/o CRA & Retrospective & NA & Linco \\
\hline Chronis et al $^{10}(\mathrm{AA})$ & 2011 & Europe & CRA & W/o CRA & Retrospective & NA & Linco \\
\hline Catalan et $\mathrm{al}^{7}$ & 2011 & Europe & Colon & Healthy & Retrospective & NA & $\mathrm{R} \& \mathrm{D}$ \\
\hline Al Khaldi et al ${ }^{12}$ & 2011 & Middle East & Colon & Healthy & Retrospective & Yes & Linco \\
\hline Yamaji et al ${ }^{22}$ (men) & 2010 & East Asia & CRA & W/o CRA & Nested & NA & NA \\
\hline Yamaji et $\mathrm{al}^{22}$ (women) & 2010 & East Asia & CRA & W/o CRA & Nested & NA & NA \\
\hline Nakajima et $\mathrm{al}^{27}$ & 2010 & East Asia & CRA & W/o CRC & Retrospective & Yes & Otsuka \\
\hline Kemik et $\mathrm{al}^{28}$ & 2010 & Middle East & Colon & Healthy & Retrospective & NA & Linco \\
\hline Gonullu et $\mathrm{al}^{33}$ & 2010 & Middle East & CRC & Healthy & Retrospective & NA & Bio-Source \\
\hline Erarslan et $\mathrm{al}^{36}$ (CRA) & 2009 & Middle East & CRA & W/o colonic polyps & Retrospective & NA & RayBiotech \\
\hline Erarslan et $\mathrm{al}^{36}$ (CRC) & 2009 & Middle East & CRC & W/o colonic polyps & Retrospective & NA & RayBiotech \\
\hline Stocks et $\mathrm{al}^{9}$ (men) & 2008 & Europe & CRC & W/o cancer & Nested & Yes & R\&D \\
\hline Stocks et al ${ }^{9}$ (women) & 2008 & Europe & CRC & W/o cancer & Nested & Yes & R\&D \\
\hline Ferroni et $\mathrm{al}^{35}$ & 2007 & Europe & CRC & W/o CRC & Retrospective & NA & BioVendor \\
\hline Wei et $\mathrm{al}^{23}$ & 2005 & USA & CRC & W/o CRC & Nested & Yes & Linco \\
\hline Otake et $\mathrm{al}^{6}$ & 2005 & East Asia & CRA & W/o colonic polyps & Retrospective & NA & Otsuka \\
\hline
\end{tabular}




\begin{tabular}{|c|c|c|c|c|c|c|c|c|c|c|}
\hline \multicolumn{2}{|c|}{ Sample size } & \multirow{2}{*}{$\begin{array}{l}\text { Sample } \\
\text { source }\end{array}$} & \multicolumn{2}{|c|}{ Adiponectin $(\mu \mathrm{g} / \mathrm{mL})$} & \multicolumn{2}{|c|}{ Age (years) } & \multicolumn{2}{|c|}{ Male gender } & \multicolumn{2}{|c|}{ BMI $\left(\mathbf{k g} / \mathbf{m}^{2}\right)$} \\
\hline Patients & Controls & & Patients & Controls & Patients & Controls & Patients & Controls & Patients & Controls \\
\hline 82 & 30 & Serum & 17.56 & 19.49 & 62.4 & 59.1 & NA & NA & NA & NA \\
\hline 52 & 30 & Serum & 15.65 & 19.49 & 62.4 & 59.1 & NA & NA & NA & NA \\
\hline 30 & 30 & Serum & 20.49 & 19.49 & 62.4 & 59.1 & NA & NA & NA & NA \\
\hline 155 & 296 & Plasma & 5.00 & 5.60 & 66.2 & 66.2 & 1.000 & 1.000 & 26.1 & 25.3 \\
\hline 152 & 297 & Plasma & 7.90 & 8.20 & 58.5 & 66.2 & 0.000 & 0.000 & 25.2 & 24.7 \\
\hline 34 & 29 & Serum & 11.28 & 6.59 & 41.3 & 66.2 & 0.588 & 0.552 & NA & NA \\
\hline 32 & 30 & Serum & 8.57 & 9.70 & 53.5 & 66.2 & 0.625 & 0.500 & 29.1 & 26.1 \\
\hline 275 & 275 & Plasma & 6.00 & 6.24 & 58.8 & 66.2 & 0.000 & 0.000 & 26.8 & 26.3 \\
\hline 217 & 650 & Plasma & 10.58 & 11.64 & 57.4 & 66.2 & 0.521 & 0.369 & 29.1 & 32.3 \\
\hline 175 & 378 & Plasma & 7.76 & 8.02 & 58.2 & 66.2 & 0.406 & $0.24 I$ & 31.7 & 32.3 \\
\hline 794 & 794 & Serum & 6.70 & 6.80 & 58.6 & 66.2 & 0.530 & 0.530 & 26.8 & 26.3 \\
\hline 466 & 466 & Serum & 6.50 & 6.80 & 58.0 & 66.2 & 0.457 & 0.457 & 26.6 & 26.4 \\
\hline 292 & 292 & Serum & 5.40 & 5.30 & 59.3 & 66.2 & 1.000 & 1.000 & 27.3 & 26.5 \\
\hline 370 & 370 & Serum & 8.30 & 8.40 & 58.6 & 66.2 & 0.000 & 0.000 & 26.4 & 26.1 \\
\hline 39 & 40 & Serum & 4.60 & 3.60 & 61.0 & 66.2 & 0.590 & 0.650 & 23.9 & 23.9 \\
\hline 270 & 519 & Plasma & 4.99 & 5.32 & 65.8 & 66.2 & 1.000 & 1.000 & 26.2 & 25.4 \\
\hline 346 & 686 & Plasma & 8.04 & 8.19 & 59.0 & 66.2 & 0.000 & 0.000 & 26.0 & 25.5 \\
\hline 21 & 26 & Plasma & 7.86 & 5.83 & 63.0 & 66.2 & 0.525 & 0.650 & 26.0 & 26.0 \\
\hline 19 & 14 & Plasma & |I.8I & 9.89 & NA & NA & NA & NA & NA & NA \\
\hline 32 & 40 & Serum & 4.80 & 6.50 & 49.5 & 40.4 & 0.563 & 0.550 & 15.9 & 21.5 \\
\hline 35 & 40 & Serum & 4.70 & 6.50 & 44.8 & 40.4 & 0.543 & 0.550 & 16.2 & 21.5 \\
\hline 457 & 834 & Plasma & 27.20 & 29.10 & NA & NA & NA & NA & NA & NA \\
\hline 42 & 30 & Serum & 14.20 & 11.80 & 65.5 & 46.0 & 1.000 & 1.000 & 27.2 & NA \\
\hline 25 & 30 & Serum & 21.90 & 22.50 & 66.0 & 44.0 & 0.000 & 0.000 & 26.4 & NA \\
\hline 27 & 40 & Serum & 9.51 & $13.9 \mid$ & 52.1 & 52.4 & 0.593 & 0.250 & 26.8 & 26.8 \\
\hline 71 & 102 & Plasma & 10.10 & 13.20 & 62.1 & 58.3 & 1.000 & 1.000 & 23.5 & 22.9 \\
\hline 94 & 102 & Plasma & 9.30 & 13.20 & 61.8 & 58.3 & 1.000 & 1.000 & 22.4 & 22.9 \\
\hline 755 & 755 & Serum & 6.71 & 6.84 & 58.6 & 58.6 & 0.542 & 0.542 & 26.9 & 26.3 \\
\hline 451 & 451 & Serum & 6.38 & 6.79 & 58.0 & 58.0 & 0.452 & 0.452 & 26.5 & 26.4 \\
\hline 31 & 30 & Serum & 5.59 & 6.38 & 55.0 & 49.0 & 1.000 & 1.000 & 24.8 & 29.1 \\
\hline 29 & 30 & Serum & 4.47 & 8.89 & 53.5 & 52.0 & 0.000 & 0.000 & 26.8 & 28.8 \\
\hline 104 & 208 & Serum & 9.45 & 10.38 & 69.8 & 69.1 & 0.625 & 0.625 & NA & NA \\
\hline 22 & 138 & Serum & 10.90 & 10.20 & 65.1 & 63.5 & 0.545 & 0.587 & 26.4 & 27.4 \\
\hline 46 & 138 & Serum & 11.20 & 10.20 & 66.2 & 63.5 & 0.565 & 0.587 & 27.1 & 27.4 \\
\hline II & 18 & Plasma & 4.07 & 8.09 & 66.0 & 44.0 & NA & NA & 26.9 & 29.3 \\
\hline 58 & 68 & Plasma & 8.60 & 4.10 & 53.0 & 60.0 & 0.500 & 0.500 & 27.9 & 26.0 \\
\hline 523 & 480 & Plasma & 3.98 & 4.37 & NA & NA & 1.000 & 1.000 & NA & NA \\
\hline 255 & 255 & Plasma & 6.81 & 7.36 & NA & NA & 0.000 & 0.000 & NA & NA \\
\hline 115 & 115 & Serum & 8.90 & 8.90 & 63.7 & 63.5 & 0.600 & 0.600 & 22.9 & 23.1 \\
\hline 126 & 36 & Serum & 4.30 & 6.50 & 43.5 & 40.4 & 0.421 & 0.444 & NA & NA \\
\hline 36 & 37 & Serum & 5.50 & 6.20 & 56.6 & 51.0 & 0.500 & $0.54 I$ & 27.2 & 27.0 \\
\hline 31 & 50 & Serum & 7.40 & 9.20 & 63.0 & 59.0 & 0.548 & 0.540 & 26.0 & 29.2 \\
\hline 23 & 50 & Serum & 7.10 & 9.20 & 57.0 & 59.0 & 0.609 & 0.540 & 24.6 & 29.2 \\
\hline 125 & 245 & Plasma & 7.00 & 6.60 & 59.8 & NA & 1.000 & 1.000 & NA & NA \\
\hline 181 & 350 & Plasma & 11.40 & 11.40 & 59.7 & NA & 0.000 & 0.000 & NA & NA \\
\hline 60 & 35 & Serum & 8.30 & 13.10 & 64.0 & 63.0 & 0.517 & 0.514 & NA & NA \\
\hline 179 & 177 & Plasma & 7.40 & 7.80 & 66.6 & 66.5 & 1.000 & 1.000 & 25.9 & 25.4 \\
\hline 51 & 52 & Plasma & 7.00 & 10.60 & 59.0 & 58.0 & 0.686 & 0.654 & 23.6 & 22.8 \\
\hline
\end{tabular}


Table I (Continued)

\begin{tabular}{|c|c|c|c|c|c|c|c|c|c|}
\hline \multirow[t]{2}{*}{ Study } & \multirow[t]{2}{*}{ Year } & \multicolumn{2}{|l|}{ WHR } & \multicolumn{2}{|l|}{ WC (cm) } & \multicolumn{2}{|l|}{ DM } & \multicolumn{2}{|c|}{ Physical activity (h/week) } \\
\hline & & Patients & Controls & Patients & Controls & Patients & Controls & Patients & Controls \\
\hline Saetang et $\mathrm{a}^{25}$ (CRC) & 2016 & NA & NA & NA & NA & NA & NA & NA & NA \\
\hline Saetang et $\mathrm{al}^{25}$ (colon) & 2016 & NA & NA & NA & NA & NA & NA & NA & NA \\
\hline Saetang et a ${ }^{25}$ (rectum) & 2016 & NA & NA & NA & NA & NA & NA & NA & NA \\
\hline Inamura et al ${ }^{29}$ (HPFS) & 2016 & NA & NA & NA & NA & NA & NA & 32.1 & 30.4 \\
\hline Inamura et $\mathrm{al}^{29}(\mathrm{NHS})$ & 2016 & NA & NA & NA & NA & NA & NA & 15.2 & 14.8 \\
\hline Zekri et al ${ }^{13}$ & 2015 & NA & NA & NA & NA & NA & NA & NA & NA \\
\hline Demir et $\mathrm{al}^{37}$ & 2015 & NA & NA & 102.8 & 95.0 & 0.000 & 0.000 & NA & NA \\
\hline Chandler et al" & 2015 & NA & NA & NA & NA & NA & NA & 15.4 & 16.5 \\
\hline Ochs-Balcom et a ${ }^{26}$ (white) & 2014 & 0.92 & 0.90 & 99.6 & 95.1 & 0.078 & 0.086 & NA & NA \\
\hline Ochs-Balcom et $\mathrm{al}^{26}(\mathrm{AA})$ & 2014 & 0.97 & 0.93 & 105.0 & 104.1 & 0.252 & 0.226 & NA & NA \\
\hline Aleksandrova et $\mathrm{al}^{5}$ (colon) & 2014 & NA & NA & 90.7 & 88.5 & NA & NA & NA & NA \\
\hline Aleksandrova et $\mathrm{al}^{5}$ (rectum) & 2014 & NA & NA & 90.4 & 89.7 & NA & NA & NA & NA \\
\hline Aleksandrova et a $\mathrm{a}^{40}$ (men) & 2014 & 0.96 & 0.94 & 97.8 & 94.8 & NA & NA & NA & NA \\
\hline Aleksandrova et $\mathrm{al}^{40}$ (women) & 2014 & 0.81 & 0.81 & 84.3 & 82.6 & NA & NA & NA & NA \\
\hline Abe Vicente et $\mathrm{al}^{43}$ & 2014 & NA & NA & NA & NA & NA & NA & NA & NA \\
\hline Song et $\mathrm{a}^{24}$ (men) & 2013 & 0.96 & 0.94 & 98.0 & 95.3 & NA & NA & 31.9 & 31.0 \\
\hline Song et $\mathrm{al}^{24}$ (women) & 2013 & 0.79 & 0.78 & 81.0 & 79.5 & NA & NA & 16.6 & 16.9 \\
\hline Danese et $\mathrm{al}^{38}$ (men) & 2013 & NA & NA & 97.5 & 95.9 & NA & NA & NA & NA \\
\hline Danese et $\mathrm{al}^{38}$ (women) & 2013 & NA & NA & 94.2 & 81.1 & NA & NA & NA & NA \\
\hline Kemik et al ${ }^{8}$ (colon) & 2012 & NA & NA & NA & NA & NA & NA & NA & NA \\
\hline Kemik et $\mathrm{al}^{8}$ (rectum) & 2012 & NA & NA & NA & NA & NA & NA & NA & NA \\
\hline Ho et $\mathrm{a}^{30}$ & 2012 & NA & NA & NA & NA & 0.000 & 0.000 & NA & NA \\
\hline Hillenbrand et $\mathrm{al}^{31}$ (men) & 2012 & NA & NA & NA & NA & NA & NA & NA & NA \\
\hline Hillenbrand et al ${ }^{31}$ (women) & 2012 & NA & NA & NA & NA & NA & NA & NA & NA \\
\hline Gulcelik et $\mathrm{al}^{32}$ & 2012 & NA & NA & NA & NA & 0.000 & 0.000 & NA & NA \\
\hline Chen et $\mathrm{al}^{39}(\mathrm{E})$ & 2012 & 0.89 & 0.86 & NA & NA & 0.000 & 0.000 & NA & NA \\
\hline Chen et $\mathrm{al}^{39}(\mathrm{~A})$ & 2012 & 0.85 & 0.86 & NA & NA & 0.000 & 0.000 & NA & NA \\
\hline Aleksandrova et $\mathrm{al}^{41}$ (colon) & 2012 & 0.88 & 0.87 & 90.4 & 88.1 & NA & NA & NA & NA \\
\hline Aleksandrova et $\mathrm{al}^{41}$ (rectum) & 2012 & 0.89 & 0.88 & 90.4 & 89.9 & NA & NA & NA & NA \\
\hline Al-Harithy and Al-Zahrani' ${ }^{42}$ (men) & 2012 & 1.23 & 0.95 & 72.6 & 75.7 & NA & NA & NA & NA \\
\hline Al-Harithy and Al-Zahrani ${ }^{42}$ (women) & 2012 & 0.95 & 1.01 & 61.0 & 84.6 & NA & NA & NA & NA \\
\hline Gialamas et $\mathrm{al}^{34}$ & 2011 & 1.01 & 0.94 & NA & NA & 0.279 & 0.173 & NA & NA \\
\hline Chronis et $\mathrm{al}^{10}$ (NOAA) & 2011 & NA & NA & 99.3 & 99.9 & 0.000 & 0.000 & NA & NA \\
\hline Chronis et $\mathrm{al}^{10}(\mathrm{AA})$ & 2011 & NA & NA & 99.2 & 99.9 & 0.000 & 0.000 & NA & NA \\
\hline Catalan et $\mathrm{al}^{7}$ & 2011 & NA & NA & NA & NA & 0.000 & 0.000 & NA & NA \\
\hline Al Khaldi et al ${ }^{12}$ & 2011 & NA & NA & 106.0 & 102.5 & 0.000 & 0.000 & NA & NA \\
\hline Yamaji et al ${ }^{22}$ (men) & 2010 & NA & NA & NA & NA & NA & NA & NA & NA \\
\hline Yamaji et al ${ }^{22}$ (women) & 2010 & NA & NA & NA & NA & NA & NA & NA & NA \\
\hline Nakajima et al ${ }^{27}$ & 2010 & NA & NA & NA & NA & NA & NA & NA & NA \\
\hline Kemik et $\mathrm{al}^{28}$ & 2010 & NA & NA & NA & NA & NA & NA & NA & NA \\
\hline Gonullu et $\mathrm{al}^{33}$ & 2010 & 0.92 & 0.93 & NA & NA & 0.000 & 0.000 & NA & NA \\
\hline Erarslan et $\mathrm{al}^{36}$ (CRA) & 2009 & NA & NA & 94.0 & 102.0 & 0.000 & 0.000 & NA & NA \\
\hline Erarslan et $\mathrm{a}^{36}(\mathrm{CRC})$ & 2009 & NA & NA & 84.0 & 102.0 & 0.000 & 0.000 & NA & NA \\
\hline Stocks et $\mathrm{al}^{9}$ (men) & 2008 & NA & NA & NA & NA & 0.000 & 0.000 & NA & NA \\
\hline Stocks et al ${ }^{9}$ (women) & 2008 & NA & NA & NA & NA & 0.000 & 0.000 & NA & NA \\
\hline Ferroni et $\mathrm{a}^{35}$ & 2007 & NA & NA & NA & NA & NA & NA & NA & NA \\
\hline Wei et $\mathrm{a}^{23}$ & 2005 & 0.95 & 0.94 & 97.0 & 95.0 & NA & NA & 28.6 & 28.9 \\
\hline Otake et $\mathrm{al}^{6}$ & 2005 & NA & NA & NA & NA & 0.000 & 0.000 & NA & NA \\
\hline
\end{tabular}

Abbreviations: ADI, adiponectin; ELISA, enzyme-linked immunosorbent assay; CRC, colorectal cancer; HPFS, Health Professionals Follow-up Study; NHS, Nurses' Health Study; CRA, colorectal adenoma; AA, advanced adenoma; NOAA, without advanced adenoma; W/o, without; BMI, body mass index; WHR, waist-hip ratio; WC, waist circumference; DM, diabetes mellitus; CRP, C-reactive protein; NA, not available. 


\begin{tabular}{|c|c|c|c|c|c|c|c|c|c|c|c|}
\hline \multicolumn{2}{|c|}{ Physical inactivity } & \multicolumn{2}{|l|}{ Smoking } & \multicolumn{2}{|c|}{ Family history } & \multicolumn{2}{|c|}{ Glucose (mmol/L) } & \multicolumn{2}{|c|}{ Insulin $(\mu \mathrm{U} / \mathrm{mL})$} & \multicolumn{2}{|c|}{ CRP (mg/L) } \\
\hline Patients & Controls & Patients & Controls & Patients & Controls & Patients & Controls & Patients & Controls & Patients & Controls \\
\hline NA & NA & NA & NA & NA & NA & NA & NA & NA & NA & NA & NA \\
\hline NA & NA & NA & NA & NA & NA & NA & NA & NA & NA & NA & NA \\
\hline NA & NA & NA & NA & NA & NA & NA & NA & NA & NA & NA & NA \\
\hline NA & NA & 0.610 & 0.550 & 0.790 & 0.860 & NA & NA & NA & NA & NA & NA \\
\hline NA & NA & 0.560 & 0.510 & 0.840 & 0.850 & NA & NA & NA & NA & NA & NA \\
\hline NA & NA & NA & NA & NA & NA & NA & NA & NA & NA & NA & NA \\
\hline NA & NA & NA & NA & NA & NA & 5.48 & 5.47 & 12.05 & 6.93 & NA & NA \\
\hline NA & NA & 0.098 & 0.113 & 0.120 & 0.098 & NA & NA & NA & NA & NA & NA \\
\hline NA & NA & 0.553 & 0.489 & 0.267 & 0.246 & 4.79 & 4.73 & 7.70 & 6.20 & 3.00 & 2.30 \\
\hline NA & NA & 0.703 & 0.608 & 0.166 & 0.238 & 5.55 & 5.22 & 15.30 & 9.90 & 2.40 & 2.30 \\
\hline 0.152 & 0.116 & 0.237 & 0.215 & NA & NA & NA & NA & NA & NA & NA & NA \\
\hline 0.146 & 0.133 & 0.281 & 0.288 & NA & NA & NA & NA & NA & NA & NA & NA \\
\hline 0.178 & 0.147 & 0.281 & 0.253 & NA & NA & NA & NA & NA & NA & 2.80 & 1.90 \\
\hline 0.127 & 0.097 & 0.200 & 0.170 & NA & NA & NA & NA & NA & NA & 3.30 & 2.70 \\
\hline NA & NA & NA & NA & NA & NA & NA & NA & NA & NA & NA & NA \\
\hline NA & NA & 0.050 & 0.049 & 0.196 & 0.139 & NA & NA & NA & NA & 1.34 & 1.13 \\
\hline NA & NA & 0.145 & 0.123 & 0.139 & 0.120 & NA & NA & NA & NA & 1.52 & 1.67 \\
\hline NA & 0.294 & NA & NA & NA & NA & 5.53 & 5.38 & NA & NA & 1.90 & 1.80 \\
\hline NA & NA & NA & NA & NA & NA & NA & NA & NA & NA & NA & NA \\
\hline NA & NA & NA & NA & NA & NA & NA & NA & NA & NA & 9.10 & 3.50 \\
\hline NA & NA & NA & NA & NA & NA & NA & NA & NA & NA & 9.10 & 3.50 \\
\hline 0.296 & 0.294 & 0.540 & 0.458 & 0.182 & 0.155 & NA & NA & 6.50 & 5.30 & NA & NA \\
\hline NA & NA & NA & NA & NA & NA & NA & NA & NA & NA & NA & NA \\
\hline NA & NA & NA & NA & NA & NA & NA & NA & NA & NA & NA & NA \\
\hline NA & NA & NA & NA & NA & NA & NA & NA & NA & NA & NA & NA \\
\hline 0.690 & 0.725 & 0.380 & 0.304 & NA & NA & NA & NA & NA & NA & NA & NA \\
\hline 0.777 & 0.725 & 0.415 & 0.304 & NA & NA & NA & NA & NA & NA & NA & NA \\
\hline 0.144 & 0.114 & 0.567 & 0.538 & NA & NA & NA & NA & NA & NA & 3.10 & 2.30 \\
\hline 0.151 & 0.131 & 0.609 & 0.603 & NA & NA & NA & NA & NA & NA & 2.40 & 2.30 \\
\hline NA & NA & NA & NA & NA & NA & NA & NA & NA & NA & NA & NA \\
\hline NA & NA & NA & NA & NA & NA & NA & NA & NA & NA & NA & NA \\
\hline 0.279 & 0.178 & 0.490 & 0.519 & NA & NA & NA & NA & NA & NA & NA & NA \\
\hline NA & NA & 0.318 & 0.304 & NA & NA & 5.34 & 5.58 & 5.80 & 5.90 & NA & NA \\
\hline NA & NA & 0.261 & 0.304 & NA & NA & 5.70 & 5.58 & 7.10 & 5.90 & NA & NA \\
\hline NA & NA & NA & NA & NA & NA & 6.06 & 5.50 & 3.80 & 12.00 & 11.96 & 4.31 \\
\hline NA & NA & NA & NA & NA & NA & 7.30 & 5.80 & 10.40 & 11.00 & NA & NA \\
\hline NA & NA & 0.138 & 0.135 & NA & NA & NA & NA & NA & NA & NA & NA \\
\hline NA & NA & 0.216 & 0.102 & NA & NA & NA & NA & NA & NA & NA & NA \\
\hline NA & NA & NA & NA & NA & NA & NA & NA & NA & NA & NA & NA \\
\hline NA & NA & NA & NA & NA & NA & NA & NA & NA & NA & 9.80 & 3.50 \\
\hline NA & NA & NA & NA & NA & NA & 5.33 & 5.15 & 10.50 & 6.10 & NA & NA \\
\hline NA & NA & NA & NA & NA & NA & 5.34 & 5.24 & 8.60 & 12.90 & NA & NA \\
\hline NA & NA & NA & NA & NA & NA & 5.12 & 5.24 & 9.70 & 12.90 & NA & NA \\
\hline NA & NA & NA & NA & NA & NA & NA & NA & NA & NA & NA & NA \\
\hline NA & NA & NA & NA & NA & NA & NA & NA & NA & NA & NA & NA \\
\hline NA & NA & NA & NA & NA & NA & NA & NA & NA & NA & NA & NA \\
\hline NA & NA & 0.051 & 0.054 & 0.207 & 0.126 & NA & NA & NA & NA & NA & NA \\
\hline NA & NA & NA & NA & NA & NA & 5.83 & 5.11 & 8.50 & 6.10 & NA & NA \\
\hline
\end{tabular}




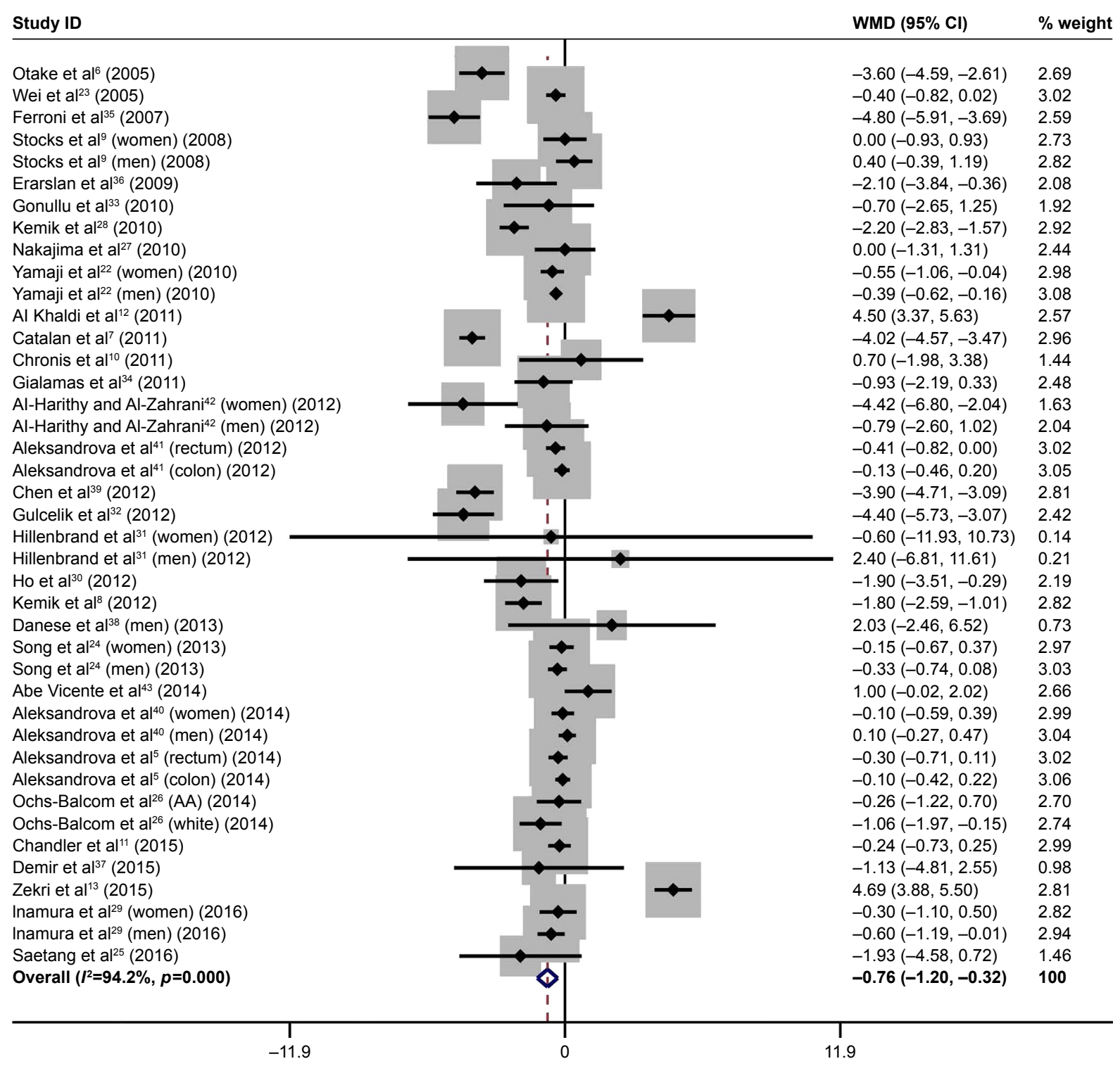

Figure I Forest plot for overall difference in total adiponectin between patients with colorectal cancer and controls.

Note: Weights are from random-effects analysis.

Abbreviations: WMD, weighted mean difference; $\mathrm{Cl}$, confidence interval.

By tumor node metastasis stage, the reduction in magnitude of total adiponectin was slightly stronger in patients with stage I-II colorectal cancer (WMD: $-1.280 \mu \mathrm{g} / \mathrm{mL}, p=0.003$ ) than in patients with stage III-IV cancer(WMD: $-1.196 \mu \mathrm{g} / \mathrm{mL}$, $p=0.005$ ), and significance was detected in both subgroups with significant heterogeneity but a low probability of publication bias.

\section{Meta-regression analysis}

Table 3 lists the results of meta-regression analysis by incorporating all characteristics to assess whether they can explain the heterogeneity between total adiponectin and colorectal cancer. Age and smoking in both patients and controls were significant potential sources of heterogeneity, and BMI, waist circumference and CRP can account for heterogeneity only in patients $(p<0.05)$.

\section{Publication bias}

Overall comparison of total adiponectin between patients with colorectal cancer and controls was not subject to publication bias, which was illustrated by both Begg's and filled funnel plots in Figure 3, as well as by the Egger's regression asymmetry test $(p=0.336)$. For comparisons in subgroup analysis, there was consistently low likelihood of publication bias across all subgroups (Egger's test, $p<0.1$ ), as presented in Table 2 . 


\begin{tabular}{|c|c|c|}
\hline Study ID & WMD $(95 \% \mathrm{Cl})$ & $\%$ weight \\
\hline \multicolumn{3}{|l|}{ HMW } \\
\hline Yamaji et al ${ }^{22}$ (men) (2010) & $-0.13(-0.31,0.05)$ & 6.82 \\
\hline Yamaji et al ${ }^{22}$ (women) (2010) & $-0.23(-0.54,0.08)$ & 6.25 \\
\hline Aleksandrova et $\mathrm{al}^{41}$ (colon) (2012) & $0.03(-0.20,0.26)$ & 6.63 \\
\hline Aleksandrova et al ${ }^{41}$ (rectum) (2012) & $-0.16(-0.45,0.13)$ & 6.36 \\
\hline Chen et al ${ }^{39}$ (2012) & $-1.60(-1.76,-1.44)$ & 6.88 \\
\hline Aleksandrova et al ${ }^{40}$ (men) (2014) & $0.00(-0.24,0.24)$ & 6.57 \\
\hline Aleksandrova et $\mathrm{al}^{40}$ (women) (2014) & $0.10(-0.26,0.46)$ & 5.97 \\
\hline Aleksandrova et al ${ }^{5}$ (colon) (2014) & $0.10(-0.12,0.32)$ & 6.64 \\
\hline Aleksandrova et al ${ }^{5}$ (rectum) (2014) & $-0.10(-0.38,0.18)$ & 6.41 \\
\hline Subtotal $\left(I^{2}=97.3 \%, p=0.000\right)$ & $-0.22(-0.70,0.25)$ & 58.52 \\
\hline \multicolumn{3}{|l|}{ Non-HMW } \\
\hline Aleksandrova et al ${ }^{41}$ (colon) (2012) & $-0.14(-0.26,-0.02)$ & 6.99 \\
\hline Aleksandrova et al ${ }^{41}$ (rectum) (2012) & $-0.35(-0.50,-0.20)$ & 6.89 \\
\hline Aleksandrova et al ${ }^{40}$ (men) (2014) & $-0.30(-0.44,-0.16)$ & 6.91 \\
\hline Aleksandrova et al ${ }^{40}$ (women) (2014) & $-0.30(-0.48,-0.12)$ & 6.80 \\
\hline Aleksandrova et al ${ }^{5}$ (colon) (2014) & $-0.20(-0.31,-0.09)$ & 6.99 \\
\hline Aleksandrova et al ${ }^{5}$ (rectum) (2014) & $-0.40(-0.55,-0.25)$ & 6.90 \\
\hline Subtotal $\left(I^{2}=52.3 \%, p=0.063\right)$ & $-0.27(-0.35,-0.19)$ & 41.48 \\
\hline Overall $\left(I^{2}=95.6 \%, p=0.000\right)$ & $-0.25(-0.47,-0.03)$ & 100 \\
\hline${ }_{-1.76}^{1}$ & 1.76 & \\
\hline
\end{tabular}

Figure 2 Forest plot for differences in two adiponectin fractions (HMW and non-HMW) between patients with colorectal cancer and controls. Note: Weights are from random-effects analysis.

Abbreviations: $\mathrm{HMW}$, high molecular weight; WMD, weighted mean difference; $\mathrm{Cl}$, confidence interval.

\section{Discussion}

The most noteworthy finding of this present study is that in line with the conclusions of most previous studies ${ }^{14-20}$ our data indicated that low total adiponectin in circulation was a significant risk factor for colorectal cancer. Moreover, for the first time, we pooled data according to adiponectin fractions and found that only non-HMW adiponectin differed significantly between patients with colorectal cancer and controls. We further identified age, gender, smoking, obesity, study design, matching status and sample size as potential sources of heterogeneity. To our knowledge, this is thus far the largest meta-analysis dealing with the relationship of circulating adiponectin and its fractions with colorectal cancer.

The connection between obesity and colorectal cancer is well established, ${ }^{44,45}$ and a great deal of supporting evidence from clinical and epidemiological data has suggested that this connection is probably mediated by abnormal adiponectin in circulation. ${ }^{46}$ Adiponectin is the most abundant hormone secreted by adipose tissue, and its anti-inflammatory and insulin-sensitizing properties may, at least in part, expound on the etiologic link between obesity and colorectal cancer. ${ }^{47,48}$
Moreover, accumulating evidence has highlighted a contributory role of adiponectin in anti-carcinogenesis. ${ }^{49,50}$ In vitro studies have showed that adiponectin can promote endothelial apoptosis, repress the maturation and proliferation of colorectal cancer cells and control colony formation, likely through the activation of AMPK-mTOR signaling pathway. ${ }^{39,51,52}$ Based on the above evidence, it is more reasonable to presume that circulating adiponectin may be involved in the pathophysiological process of colorectal cancer.

Consistent with the findings of major published studies, ${ }^{14-20}$ we confirmed in this present study that low total adiponectin in circulation was a significant risk factor for colorectal cancer. As with most previous meta-analyses, heterogeneity is a serious issue that limits interpretation of effect estimates and requires careful exploration..$^{53}$ As a secondary goal of this study, we employed both subgroup analysis and meta-regression analysis to seek possible causes of heterogeneity between studies. In particular, the reduction in total adiponectin was more obvious for CRA and colon cancer, and in studies enrolling subjects of male gender, from East Asia and with normal weight. In addition, the association of total adiponectin with colorectal 
Table 2 Subgroup analysis of the difference in circulating adiponectin between patients with colorectal cancer and controls

\begin{tabular}{|c|c|c|c|c|c|c|c|c|}
\hline Characteristics & Subgroups & $\begin{array}{l}\text { Studies } \\
\text { (number) }\end{array}$ & WMD & $95 \% \mathrm{Cl}$ & $p^{D}$ & $I^{2}$ & $p^{H}$ & $p^{\mathrm{E}}$ \\
\hline \multirow[t]{4}{*}{ Cancer subtype } & CRC & 20 & -0.736 & -1.519 to 0.047 & 0.065 & $94.0 \%$ & $<0.001$ & 0.514 \\
\hline & CRA & 10 & -0.892 & -1.573 to -0.211 & 0.010 & $80.1 \%$ & $<0.001$ & 0.473 \\
\hline & Colon & 10 & -1.073 & -2.095 to -0.050 & 0.040 & $97.3 \%$ & $<0.00 \mathrm{I}$ & 0.403 \\
\hline & Rectum & 4 & $-0.68 I$ & -1.358 to -0.005 & 0.048 & $75.4 \%$ & 0.007 & 0.778 \\
\hline \multirow[t]{4}{*}{ Region } & East Asia & 6 & -1.707 & -2.979 to -0.436 & 0.008 & $95.1 \%$ & $<0.001$ & 0.213 \\
\hline & America & 10 & -0.343 & -0.599 to -0.087 & 0.009 & $36.7 \%$ & 0.115 & 0.683 \\
\hline & Europe & 15 & -0.749 & $-1.45 \mid$ to -0.047 & 0.037 & $94.3 \%$ & $<0.001$ & 0.541 \\
\hline & Middle East & 10 & -0.787 & -3.015 to 1.442 & 0.489 & $97.1 \%$ & $<0.001$ & 0.787 \\
\hline \multirow[t]{2}{*}{ Study design } & Retrospective & 22 & -1.179 & -2.536 to 0.177 & 0.088 & $96.2 \%$ & $<0.001$ & 0.489 \\
\hline & Nested & 19 & -0.266 & -0.380 to -0.152 & $<0.001$ & $12.4 \%$ & 0.303 & 0.409 \\
\hline \multirow[t]{2}{*}{ Matching status } & NA & 21 & -1.472 & -2.452 to -0.49 I & 0.003 & $96.1 \%$ & $<0.00 \mathrm{I}$ & $0.4 I I$ \\
\hline & Yes & 20 & -0.099 & -0.389 to 0.191 & 0.504 & $79.0 \%$ & $<0.001$ & 0.693 \\
\hline \multirow[t]{2}{*}{ Sample source } & Plasma & 18 & $-0.7 \mid 4$ & -1.358 to -0.070 & 0.030 & $95.1 \%$ & $<0.001$ & 0.783 \\
\hline & Serum & 23 & -0.803 & -1.430 to -0.176 & 0.012 & $93.3 \%$ & $<0.001$ & 0.303 \\
\hline \multirow[t]{2}{*}{ Sample size (median) } & $\leq 230$ & 21 & -1.189 & -2.609 to 0.231 & 0.101 & $96.4 \%$ & $<0.00 \mathrm{I}$ & $0.51 I$ \\
\hline & $>230$ & 20 & -0.271 & -0.385 to -0.157 & $<0.001$ & $12.1 \%$ & 0.304 & 0.262 \\
\hline \multirow[t]{2}{*}{ Gender } & Men & 9 & -0.671 & -1.255 to -0.086 & 0.025 & $90.4 \%$ & $<0.001$ & 0.536 \\
\hline & Women & 8 & -0.329 & -0.693 to 0.035 & 0.077 & $49.6 \%$ & $<0.001$ & 0.206 \\
\hline \multirow[t]{2}{*}{ BMI } & $<25 \mathrm{~kg} / \mathrm{m}^{2}$ & 7 & -1.627 & -3.092 to -0.163 & 0.029 & $92.1 \%$ & $<0.001$ & 0.434 \\
\hline & $\geq 25 \mathrm{~kg} / \mathrm{m}^{2}$ & 24 & -0.527 & -1.016 to -0.039 & 0.034 & $92.4 \%$ & $<0.001$ & 0.611 \\
\hline \multirow[t]{2}{*}{ WHR } & $<0.9 \mathrm{~m}$ & 5 & -0.878 & -1.798 to 0.043 & 0.062 & $94.7 \%$ & $<0.001$ & 0.112 \\
\hline & $\geq 0.9 \mathrm{~m}$ & 9 & -0.522 & -0.939 to -0.106 & 0.014 & $60.6 \%$ & 0.009 & 0.496 \\
\hline DM & Without & 12 & -1.385 & -3.003 to 0.233 & 0.093 & $96.1 \%$ & $<0.001$ & 0.349 \\
\hline \multirow[t]{2}{*}{ TNM stage } & I-II & 9 & -1.280 & -2.115 to -0.446 & 0.003 & $90.1 \%$ & $<0.001$ & 0.103 \\
\hline & III-IV & 9 & -1.196 & -2.033 to -0.358 & 0.005 & $92.6 \%$ & $<0.001$ & 0.219 \\
\hline
\end{tabular}

Notes: $p^{\mathrm{D}}$, the $p$-value for WMD; $l^{2}$, inconsistency index; $p^{\mathrm{H}}$, the $p$-value for heterogeneity; $p^{\mathrm{E}}$, the $p$-value for Egger's regression asymmetry test.

Abbreviations: WMD, weighted mean difference; $\mathrm{Cl}$, confidence interval; CRC, colorectal cancer; CRA, colorectal adenoma; BMI, body mass index; WHR, waist-hip ratio; DM, diabetes mellitus; TNM, tumor node metastasis; NA, not available.

cancer was markedly significant in studies with a nested casecontrol design and a large sample size, relative to studies with a retrospective case-control design and a small sample size, indicating the robustness of our observation. Colorectal cancer is a highly heterogeneous disease to which environmental exposure, germ-line susceptibility determinants and accumulated genetic and epigenetic changes contribute interactively. ${ }^{54}$ For example, cigarette smoking and alcohol drinking, which are more prevalent in men than in women, are established risk factors for the development and progression of colorectal

Table 3 The meta-regression analysis of all characteristics in both patients with colorectal cancer and controls

\begin{tabular}{|c|c|c|c|c|c|}
\hline \multirow[t]{2}{*}{ Characteristics } & \multirow{2}{*}{$\begin{array}{l}\text { Studies } \\
\text { (number) }\end{array}$} & \multicolumn{2}{|l|}{ Patients } & \multicolumn{2}{|l|}{ Controls } \\
\hline & & Coefficient & $p$-value & Coefficient & $p$-value \\
\hline Age & 36 & -0.21 & 0.012 & 0.18 & 0.019 \\
\hline Gender & 38 & -8.45 & 0.076 & 8.37 & 0.073 \\
\hline BMI & 29 & 0.41 & 0.039 & -0.35 & 0.091 \\
\hline WHR & 14 & 5.04 & 0.387 & -10.47 & 0.282 \\
\hline WC & 19 & 0.14 & 0.008 & -0.09 & 0.177 \\
\hline DM & 15 & $-3.7 \mathrm{I}$ & 0.913 & 8.86 & 0.840 \\
\hline Physical activity & 6 & -0.05 & 0.785 & 0.04 & 0.842 \\
\hline Physical inactivity & 9 & 1.46 & 0.836 & -7.74 & 0.315 \\
\hline Smoking & 20 & -10.59 & 0.032 & 10.59 & 0.043 \\
\hline Family history & 9 & -0.66 & 0.842 & 0.34 & 0.909 \\
\hline Glucose & 10 & 0.93 & 0.640 & 2.37 & 0.609 \\
\hline Insulin & 10 & 0.28 & 0.316 & 0.03 & 0.930 \\
\hline CRP & 12 & -0.37 & 0.015 & 0.20 & 0.698 \\
\hline
\end{tabular}

Abbreviations: BMI, body mass index; WHR, waist-hip ratio; WC, waist circumference; DM, diabetes mellitus; CRP, C-reactive protein. 

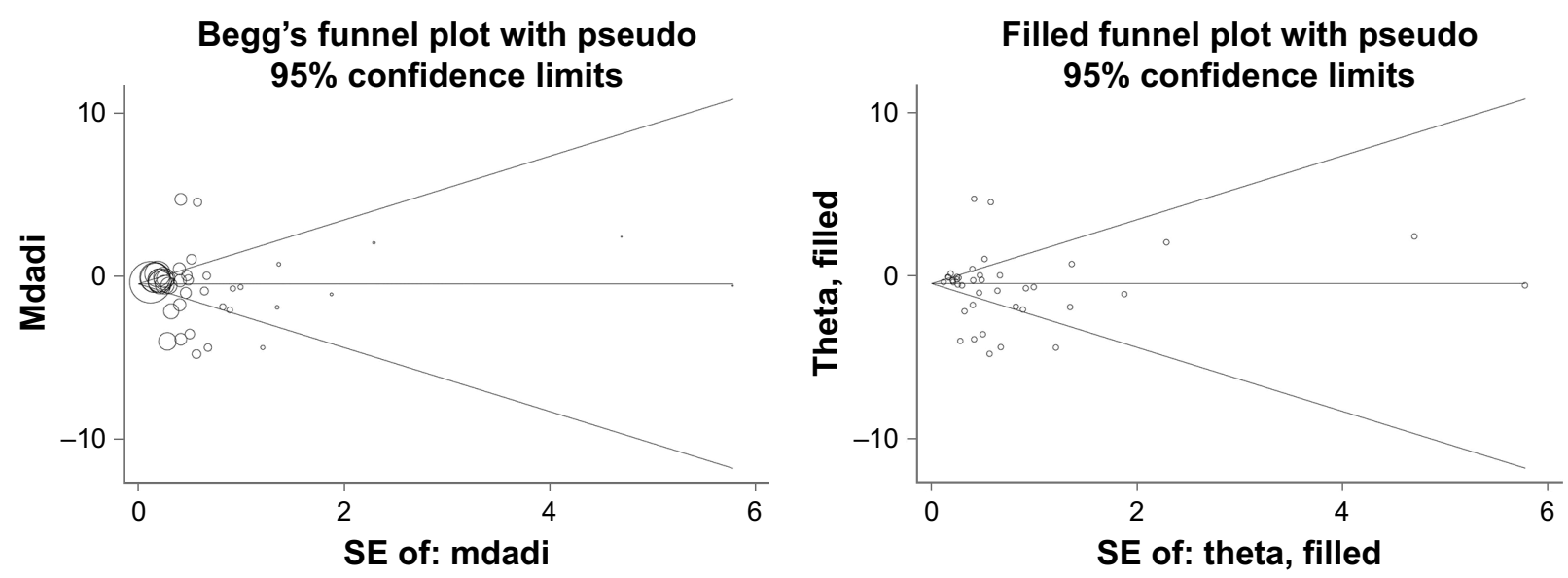

Figure 3 Begg's and filled funnel plots for difference in circulating total adiponectin between patients with colorectal cancer and controls. Abbreviations: mdadi, mean value of total adiponectin; SE, standard error.

cancer, ${ }^{55,56}$ which might serve as a possible interpretation for gender-specific difference in adiponectin observed in the present study. Nevertheless, even though we have made great endeavors to seek causes of heterogeneity, there is still strong evidence of heterogeneity in some subgroups, indicating that residual confounding from other sources of heterogeneity in colorectal cancer risk is likely. We agree that further explorations are necessary, and especially analysis of individual participant data could yield further insights.

However, a growing body of research has reported diverse biological activities of different adiponectin fractions, mainly focusing on HMW (insulin sensitivity) and non-HMW (inflammation response) forms. ${ }^{5,24}$ In this context, the sufficient number of eligible studies in this meta-analysis enables us to investigate different adiponectin fractions, and our findings revealed that low non-HMW adiponectin in circulation was a significant risk factor for colorectal cancer and there was no observable significant difference for HMW adiponectin. As previously discussed, non-HMW adiponectin plays a major role in the inflammatory process. ${ }^{24}$ Systemic inflammation is a key manifestation of cancer progression and metastasis in many types of cancer including colorectal cancer. ${ }^{57}$ We thus develop a further presumption that nonHMW adiponectin may be involved in colorectal carcinogenesis by regulating inflammatory responses. Addressing this presumption is beyond the scope of this meta-analysis, and further experimental studies are required.

Finally, the present study needs to be interpreted cautiously, bearing in mind the following limitations. First, we only retrieved the literature for the papers published in English language, and selection bias cannot be ruled out. Second, all involved studies in this meta-analysis were observational in nature, either cross-sectional or nested, which hindered further causality inference. Third, as discussed above, sources of heterogeneity for a majority of comparisons were not fully accounted for, and additional considerations were necessary. For example, low circulating adiponectin was found to be associated with $K R A S$-mutant colorectal cancer risk but not with $K R A S$-wild-type cancer risk. ${ }^{29}$ Fourth, only data on circulating plasma/serum adiponectin were summarized, and it is expected that tissue adiponectin concentration in tumor environment may be more relevant to the evaluation of colorectal cancer risk than its circulating concentration. However, data on tissue adiponectin are rarely reported. Fifth, circulating adiponectin concentration was affected by medical treatment or drug intervention, which cannot be taken into account because of lack of data.

In conclusion, through a comprehensive meta-analysis of 7,554 patients with colorectal cancer and 9,798 controls, our data indicate that low total adiponectin, especially its non-HMW fraction, represents a promising risk factor for colorectal cancer. Although there is still residual confounding unaccounted for, we believe that this study can aid in better understanding cancer heterogeneity, highlighting the importance of anti-inflammation therapies to prevent or delay the occurrence of colorectal cancer and thereby providing new insight in its physiology. In addition, further studies are needed to explore underlying mechanisms.

\section{Author contributions}

WL and $\mathrm{ZH}$ searched the literature and identified potential papers, extracted the data, performed statistical analysis and drafted the manuscript. NL checked the data and results. HL designed the study and polished the language of the manuscript. All authors contributed toward data analysis, drafting and critically revising the paper, gave final approval 
of the version to be published, and agree to be accountable for all aspects of the work.

\section{Disclosure}

The authors report no conflicts of interest in this work.

\section{References}

1. Heiker JT, Kosel D, Beck-Sickinger AG. Molecular mechanisms of signal transduction via adiponectin and adiponectin receptors. Biol Chem. 2010;391(9):1005-1018.

2. Achari AE, Jain SK. Adiponectin, a therapeutic target for obesity, diabetes, and endothelial dysfunction. Int J Mol Sci. 2017;18(6):pii E1321.

3. Diez JJ, Iglesias P. The role of the novel adipocyte-derived hormone adiponectin in human disease. Eur J Endocrinol. 2003;148(3):293-300.

4. Liu M, Liu F. Regulation of adiponectin multimerization, signaling and function. Best Pract Res Clin Endocrinol Metab. 2014;28(1):25-31.

5. Aleksandrova K, Jenab M, Bueno-de-Mesquita HB, et al. Biomarker patterns of inflammatory and metabolic pathways are associated with risk of colorectal cancer: results from the European Prospective Investigation into Cancer and Nutrition (EPIC). Eur J Epidemiol. 2014;29(4): 261-275.

6. Otake S, Takeda H, Suzuki Y, et al. Association of visceral fat accumulation and plasma adiponectin with colorectal adenoma: evidence for participation of insulin resistance. Clin Cancer Res. 2005;11(10): $3642-3646$

7. Catalan V, Gomez-Ambrosi J, Rodriguez A, et al. Up-regulation of the novel proinflammatory adipokines lipocalin-2, chitinase-3 like-1 and osteopontin as well as angiogenic-related factors in visceral adipose tissue of patients with colon cancer. J Nutr Biochem. 2011;22(7): 634-641.

8. Kemik O, Kemik AS, Begenik H, et al. The relationship among acutephase response proteins, cytokines, and hormones in various gastrointestinal cancer types patients with cachectic. Hum Exp Toxicol. 2012; 31(2):117-125.

9. Stocks T, Lukanova A, Johansson M, et al. Components of the metabolic syndrome and colorectal cancer risk; a prospective study. Int $J$ Obes (Lond). 2008;32(2):304-314.

10. Chronis A, Thomopoulos K, Sapountzis A, et al. Adiposity factors are not related to the presence of colorectal adenomas. Clin Exp Gastroenterol. 2011;4:257-261.

11. Chandler PD, Buring JE, Manson JE, et al. Association between plasma adiponectin levels and colorectal cancer risk in women. Cancer Causes Control. 2015;26(7):1047-1052.

12. Al Khaldi RM, Al Mulla F, Al Awadhi S, Kapila K, Mojiminiyi OA. Associations of single nucleotide polymorphisms in the adiponectin gene with adiponectin levels and cardio-metabolic risk factors in patients with cancer. Dis Markers. 2011;30(4):197-212.

13. Zekri AR, Bakr YM, Ezzat MM, Zakaria MS, Elbaz TM. Circulating levels of adipocytokines as potential biomarkers for early detection of colorectal carcinoma in Egyptian patients. Asian Pac J Cancer Prev. 2015;16(16):6923-6928.

14. Pei Y, Xu Y, Niu W. Causal relevance of circulating adiponectin with cancer: a meta-analysis implementing Mendelian randomization. Tumour Biol. 2015;36(2):585-594.

15. Joshi RK, Lee SA. Obesity related adipokines and colorectal cancer: a review and meta-analysis. Asian Pac J Cancer Prev. 2014;15(1): 397-405.

16. Joshi RK, Kim WJ, Lee SA. Association between obesity-related adipokines and colorectal cancer: a case-control study and meta-analysis. World J Gastroenterol. 2014;20(24):7941-7949.

17. Perrier $\mathrm{S}$, Jarde $\mathrm{T}$. Adiponectin, an anti-carcinogenic hormone? A systematic review on breast, colorectal, liver and prostate cancer. Curr Med Chem. 2012;19(32):5501-5512.
18. Izadi V, Farabad E, Azadbakht L. Serum adiponectin level and different kinds of cancer: a review of recent evidence. ISRN Oncol. 2012; 2012:982769.

19. An W, Bai Y, Deng SX, et al. Adiponectin levels in patients with colorectal cancer and adenoma: a meta-analysis. Eur J Cancer Prev. 2012; 21(2):126-133.

20. Xu XT, Xu Q, Tong JL, et al. Meta-analysis: circulating adiponectin levels and risk of colorectal cancer and adenoma. J Dig Dis. 2011; 12(4):234-244.

21. Moher D, Liberati A, Tetzlaff J, Altman DG; PRISMA Group. Preferred reporting items for systematic reviews and meta-analyses: the PRISMA statement. BMJ. 2009;339:b2535.

22. Yamaji T, Iwasaki M, Sasazuki S, Tsugane S. Interaction between adiponectin and leptin influences the risk of colorectal adenoma. Cancer Res. 2010;70(13):5430-5437.

23. Wei EK, Giovannucci E, Fuchs CS, Willett WC, Mantzoros CS. Low plasma adiponectin levels and risk of colorectal cancer in men: a prospective study. J Natl Cancer Inst. 2005;97(22):1688-1694.

24. Song M, Zhang X, Wu K, et al. Plasma adiponectin and soluble leptin receptor and risk of colorectal cancer: a prospective study. Cancer Prev Res (Phila). 2013;6(9):875-885.

25. Saetang N, Boonpipattanapong T, Palanusont A, Maneechay W, Sangkhathat S. Alteration of leptin and adiponectin in multistep colorectal tumorigenesis. Asian Pac J Cancer Prev. 2016;17(4):2119-2123.

26. Ochs-Balcom HM, Cannioto R, Nie J, et al. Adipokines do not mediate the association of obesity and colorectal adenoma. J Cancer Epidemiol. 2014;2014:371254.

27. Nakajima TE, Yamada Y, Hamano T, et al. Adipocytokines as new promising markers of colorectal tumors: adiponectin for colorectal adenoma, and resistin and visfatin for colorectal cancer. Cancer Sci. 2010;101(5):1286-1291.

28. Kemik O, Sumer A, Kemik AS, et al. The relationship among acutephase response proteins, cytokines and hormones in cachectic patients with colon cancer. World J Surg Oncol. 2010;8:85.

29. Inamura $\mathrm{K}$, Song $\mathrm{M}$, Jung $\mathrm{S}$, et al. Prediagnosis plasma adiponectin in relation to colorectal cancer risk according to KRAS mutation status. J Natl Cancer Inst. 2016;108(4):pii djv363.

30. Ho GY, Wang T, Gunter MJ, et al. Adipokines linking obesity with colorectal cancer risk in postmenopausal women. Cancer Res. 2012; 72(12):3029-3037.

31. Hillenbrand A, Fassler J, Huber N, et al. Changed adipocytokine concentrations in colorectal tumor patients and morbidly obese patients compared to healthy controls. BMC Cancer. 2012;12:545.

32. Gulcelik MA, Colakoglu K, Dincer H, Dogan L, Yenidogan E, Gulcelik NE. Associations between adiponectin and two different cancers: breast and colon. Asian Pac J Cancer Prev. 2012;13(1): 395-398.

33. Gonullu G, Kahraman H, Bedir A, Bektas A, Yucel I. Association between adiponectin, resistin, insulin resistance, and colorectal tumors. Int J Colorectal Dis. 2010;25(2):205-212.

34. Gialamas SP, Petridou ET, Tseleni-Balafouta S, et al. Serum adiponectin levels and tissue expression of adiponectin receptors are associated with risk, stage, and grade of colorectal cancer. Metabolism. 2011;60(11):1530-1538.

35. Ferroni P, Palmirotta R, Spila A, et al. Prognostic significance of adiponectin levels in non-metastatic colorectal cancer. Anticancer Res. 2007;27(1B):483-489.

36. Erarslan E, Turkay C, Koktener A, Koca C, Uz B, Bavbek N. Association of visceral fat accumulation and adiponectin levels with colorectal neoplasia. Dig Dis Sci. 2009;54(4):862-868.

37. Demir N, Ahishali E, Dolapcioglu C, et al. The relationship between serum adiponectin and resistin levels, insulin resistance and colorectal adenomas. Turk J Gastroenterol. 2015;26(1):20-24.

38. Danese E, Minicozzi AM, Montagnana M, De Manzoni G, Lippi G, Guidi GC. Lack of an association between circulating adiponectin levels and risk of colorectal adenoma. Clin Lab. 2013;59(1-2):211-214. 
39. Chen MW, Ye S, Zhao LL, et al. Association of plasma total and highmolecular-weight adiponectin with risk of colorectal cancer: an observational study in Chinese male. Med Oncol. 2012;29(5):3129-3135.

40. Aleksandrova K, Drogan D, Boeing H, et al. Adiposity, mediating biomarkers and risk of colon cancer in the European prospective investigation into cancer and nutrition study. Int J Cancer. 2014;134(3): 612-621.

41. Aleksandrova K, Boeing H, Jenab M, et al. Total and high-molecular weight adiponectin and risk of colorectal cancer: the European Prospective Investigation into Cancer and Nutrition Study. Carcinogenesis. 2012;33(6):1211-1218.

42. Al-Harithy RN, Al-Zahrani MH. The adiponectin gene, ADIPOQ, and genetic susceptibility to colon cancer. Oncol Lett. 2012;3(1): $176-180$.

43. Abe Vicente M, Donizetti Silva T, Barao K, Vitor Felipe A, Oyama Missae L, Manoukian Forones N. The influence of nutritional status and disease on adiponectin and TNF-alpha; levels in colorectal cancer patients. Nutr Hosp. 2014;30(1):140-146.

44. Billy M, Sholihah H, Andanni K, Anggraeni MI, Siregar SM, Mirtha LT. Obesity as predictor of mortality of colorectal cancer: an evidence-based case report. Acta Med Indones. 2016;48(3):242-246.

45. Kasi PM, Zafar SY, Grothey A. Is obesity an advantage in patients with colorectal cancer? Expert Rev Gastroenterol Hepatol. 2015;9(11): 1339-1342.

46. La Cava A. Adiponectin: a relevant player in obesity-related colorectal cancer? Gut. 2013;62(4):483-484.

47. Otani K, Ishihara S, Yamaguchi $\mathrm{H}$, et al. Adiponectin and colorectal cancer. Surg Today. 2017;47(2):151-158.
48. Yamamoto H, Maeda K, Arima H, et al. Perioperative adiponectin measurement is useful for prediction of postoperative infection in patients with colorectal cancer. Ann Surg Oncol. 2016;23(Suppl 4):540-545.

49. Tae CH, Kim SE, Jung SA, et al. Involvement of adiponectin in early stage of colorectal carcinogenesis. BMC Cancer. 2014;14:811.

50. Kapoor S. Adiponectin and its role in gastrointestinal carcinogenesis. Am J Gastroenterol. 2009;104(1):243-244; author reply 244-245.

51. Sugiyama M, Takahashi H, Hosono K, et al. Adiponectin inhibits colorectal cancer cell growth through the AMPK/mTOR pathway. Int J Oncol. 2009;34(2):339-344.

52. Kim AY, Lee YS, Kim KH, et al. Adiponectin represses colon cancer cell proliferation via AdipoR1- and -R2-mediated AMPK activation. Mol Endocrinol. 2010;24(7):1441-1452.

53. Petitti DB. Approaches to heterogeneity in meta-analysis. Stat Med. 2001;20(23):3625-3633.

54. Takayama T, Miyanishi K, Hayashi T, Sato Y, Niitsu Y. Colorectal cancer: genetics of development and metastasis. $J$ Gastroenterol. 2006;41(3):185-192.

55. Cho S, Shin A, Park SK, Shin HR, Chang SH, Yoo KY. Alcohol drinking, cigarette smoking and risk of colorectal cancer in the Korean multicenter cancer cohort. J Cancer Prev. 2015;20(2):147-152.

56. Hashibe M, Hunt J, Wei M, Buys S, Gren L, Lee YC. Tobacco, alcohol, body mass index, physical activity, and the risk of head and neck cancer in the prostate, lung, colorectal, and ovarian (PLCO) cohort. Head Neck. 2013;35(7):914-922.

57. Rossi S, Basso M, Strippoli A, et al. Are markers of systemic inflammation good prognostic indicators in colorectal cancer? Clin Colorectal Cancer. 2017;16(4):264-274.
OncoTargets and Therapy

\section{Publish your work in this journal}

OncoTargets and Therapy is an international, peer-reviewed, open access journal focusing on the pathological basis of all cancers, potential targets for therapy and treatment protocols employed to improve the management of cancer patients. The journal also focuses on the impact of management programs and new therapeutic agents and protocols on

\section{Dovepress}

patient perspectives such as quality of life, adherence and satisfaction. The manuscript management system is completely online and includes a very quick and fair peer-review system, which is all easy to use. Visit http://www.dovepress.com/testimonials.php to read real quotes from published authors. 